\title{
O TRATAMENTO DIFERENCIADO DOS PAÍSES EM DESENVOLVIMENTO NO DIREITO INTERNACIONAL AMBIENTAL: PERSPECTIVAS A PARTIR DO ACORDO DE PARIS
}

\author{
DIFFERENTIATED TREATMENT OF DEVELOPING COUNTRIES IN INTERNATIONAL \\ ENVIRONMENTAL LAW: PERSPECTIVES FROM THE PARIS AGREEMENT
}

\section{EL TRATAMIENTO DIFERENCIADO DE LOS PAÍSES EN DESARROLLO EN EL DERECHO INTERNACIONAL AMBIENTAL: PERSPECTIVAS A PARTIR DEL ACUERDO DE PARÍS}

\section{André Soares Oliveira ${ }^{1}$}

Licença CC BY:

Artigo distribuído sob os termos Creative Commons, permite uso e distribuição irrestrita em qualquer meio desde que o autor credite a fonte original.

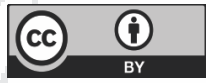

\begin{abstract}
Resumo: O tratamento diferenciado desafia a igualdade soberana do direito internacional, vista como injusta pelos países em desenvolvimento. O princípio das responsabilidades comuns mas diferenciadas e respectivas capacidades expressa esse tratamento diferenciado no direito internacional ambiental. Objetiva-se analisar a instrumentalização deste princípio no Acordo de Paris da Convenção-Quadro das Nações Unidas sobre Mudanças Climáticas. A pesquisa vale-se do método dedutivo, uma abordagem estruturalista e materialista-histórica para análise dos textos jurídicos. Deixando para trás a lógica descendente do Protocolo de Quioto, lastreando por compromissos internacionalmente firmados, o Acordo de Paris, sob a alegação de prover diferenciação para todos, opta por um modelo ascendente por meio de contribuições nacionalmente determinadas. O Acordo de Paris deixa de lado os conflitos distributivos do princípio das responsabilidades comuns mas diferenciadas e respectivas capacidades, enfraquecendo a ação política como bloco dos países em desenvolvimento para assegurarem níveis adequados de ambição no enfrentamento das mudanças climáticas.
\end{abstract}

Palavras-chave: tratamento diferenciado dos países em desenvolvimento; mudanças climáticas; multilateralismo redistributivo; acordo de paris; contribuições nacionalmente determinadas.

Abstract: Differential treatment challenges the sovereign equality of international law, which is seen as unfair by developing countries. The principle of common but differentiated responsibilities and respective capabilities expresses this differential treatment in international environmental law. The aim is to analyze the use of this principle in the Paris Agreement of the United Nations Framework Convention on Climate Change. The research is based on the deductive method, in a structuralist and materialist-historical approach to the analysis of legal texts. Leaving behind the downward logic of the Kyoto Protocol, backed by internationally agreed commitments, the Paris Agreement, on the grounds of providing differentiation for all, opts for a bottom-

1 Doutor em Direito pela Universidade Federal do Rio Grande do Sul (UFRGS), Mestre em Direito pela Universidade Federal de Santa Catarina (UFSC) e Bacharel em Ciências Jurídicas e Sociais pela Universidade Federal de Campina Grande (UFCG). Professor da Faculdade Paraíso do Ceará - FAPCE. Líder do Grupo de Pesquisa 'Constitucionalismo Contemporâneo e Democracia' (CNPq/FAPCE). E-mail: asoliveira3@ gmail.com. 
up model through nationally determined contributions. The Paris Agreement leaves aside the distributive conflicts of the principle of common but differentiated responsibilities and respective capabilities, weakening political action as a bloc of developing countries to ensure adequate levels of ambition in tackling climate change.

Keywords: differentiated treatment of developing countries; climate changes; redistributive multilateralism; Paris agreement; nationally determined contributions.

Resumen: El tratamiento diferenciado afronta la igualdad soberana del derecho internacional, vista como injusta por los países en desarrollo. El principio de las responsabilidades comunes, pero diferenciadas y respectivas capacidades expresa este tratamiento diferenciado en el derecho internacional ambiental. Se objetiva analizar el instrumento de este principio en el Acuerdo de París en la Convención de las Naciones Unidas sobre Cambios Climáticos. La investigación se vale del método deductivo, un abordaje estructuralista y materialista histórica para análisis de textos jurídicos. Dejando para después la lógica descendiente del Protocolo de Quioto, respaldado por compromisos internacionalmente firmados, el Acuerdo de París, bajo el alegato de proporcionar diferenciación para todos, opta por un modelo ascendente por medio de contribuciones nacionalmente determinadas. El Acuerdo de París deja de lado los conflictos distributivos del principio de responsabilidades comunes, pero diferenciadas y respectivas capacidades, debilitando la acción política como bloque de los países en desarrollo para asegurar niveles adecuados de ambición al enfrentamiento de los cambios climáticos.

Palabras clave: tratamiento diferenciado de los países en desarrollo; cambios climáticos; multilateralismo redistributivo; acuerdo de París; contribuciones nacionalmente determinadas.

\section{INTRODUÇÃO}

A desigualdade Norte-Sul é elemento fundamental da política internacional desde a Segunda Guerra Mundial, quando a pauta é desenvolvimento. Primeiramente no âmbito da Nova Ordem Econômica Internacional, que preconizou o tratamento diferenciado dos países em desenvolvimento, logo tal debate se fez presente na política ambiental global, na Conferência das Nações Unidas sobre o Meio Ambiente Humano.

O tratamento diferenciado dos países em desenvolvimento, no âmbito do direito internacional ambiental, materializa-se no princípio das responsabilidades comuns mas diferenciadas e respectivas capacidades. A partir deste princípio, busca-se implementar soluções justas no enfrentamento dos problemas ambientais, sacrificando-se a noção de igualdade soberana.

A Convenção-Quadro das Nações Unidas sobre Mudanças Climáticas (CQMC), de 1992, erige o princípio como um dos fundamentos da governança global do clima, que chega ao auge no Protocolo de Quioto, em 1997, formado por compromissos internacionalmente negociados de redução de emissões para um determinado conjunto de países. Esse documento foi posteriormente acusado de desestimular a participação de grandes poluidores, como os Estados Unidos e de não abarcar novos grandes poluidores, como a China, a Índia e mesmo o próprio Brasil. Essas dissensões levaram a um conjunto de planos de ação e acordos que conduziram ao Acordo de Paris, celebrado em 2016, e destinado a substituir o Protocolo por meio de uma arquitetura jurídica que lhe é oposta. 
Valendo-se do método dedutivo e uma abordagem estruturalista por meio de técnicas de pesquisa bibliográfica e documental, essa pesquisa questiona criticamente a forma como o Acordo de Paris lida com o princípio das responsabilidades comuns mas diferenciadas e respectivas capacidades - repita-se: fundamento da CQMC a qual ele está submetido - tendo em vista a persistência, ainda que de forma dinâmica, da desigualdade Norte-Sul.

A hipótese central é que, de fato, o tratamento diferenciado dos países em desenvolvimento não pode estagnar-se às fórmulas jurídicas de diferenciação erigidas décadas atrás e que novas formas de diferenciação em um mundo cada vez mais complexo e dinâmico devem ser consideradas. Porém, não se pode perder de vista que tal tratamento diferenciado surge para o enfrentamento da desigualdade Norte-Sul, cuja doutrina do desenvolvimento, em suas diversas variações, não conseguiu equalizar. A fim de desenvolver adequadamente o tema, em um primeiro momento, procura-se evidenciar os fundamentos do princípio das responsabilidades comuns mas diferenciadas e respectivas capacidades, assim como ocorre com sua operacionalização nas obrigações de um tratado. Na segunda parte, será analisado e debatido como o Acordo de Paris trata o princípio das responsabilidades comuns mas diferenciadas e respectivas capacidades, eixo da CQMC, assim como as perspectivas que o mencionado Acordo assinala para os países em desenvolvimento.

\section{1 - O TRATAMENTO DIFERENCIADO DOS PAÍSES EM DESENVOLVIMENTO NO DIREITO INTERNACIONAL AMBIENTAL: ENTRE A RESPONSABILIDADE HISTÓRICA E A CAPACIDADE TÉCNICA}

O adensamento das relações internacionais depois da Segunda Guerra Mundial evidenciou problemas que demandam uma ação coletiva permanente, de modo que o apego estrito à soberania se torna um empecilho para o desenvolvimento de relações de solidariedade entre os Estados e mesmo a emergência de instituições internacionais robustas. ${ }^{2}$

Em um contexto marcado pelo fosso Norte-Sul e pela paralisia do Conselho de Segurança, devido à Guerra Fria, o Terceiro Mundo, como bloco, cresce na Assembleia Geral das Nações Unidas, onde emplaca novas formulações jurídicas que lhes sejam favoráveis. Entre os mecanismos, encontra-se o tratamento diferenciado aos países em desenvolvimento, cuja primeira e mais completa expressão normativa foi a Nova Ordem Econômica Internacional (NOEI). De fato, desde 1974, praticamente todos os tratados multilaterais têm levado em conta a necessidade dos países em desenvolvimento e mesmo feito especificações em relação a eles. ${ }^{3}$

2 CULLET, P. Differential treatment in international law: towards a new paradigm of inter-state relations. European Journal Of International Law, [s.I], v. 10, n. 3, p.549-582, 1 mar. 1999. Oxford University Press (OUP). DOI: 10.1093/ejil/10.3.549.

3 MAGRAW, Daniel Barstow. Legal treatment of developing countries. Colorado Journal of International Environmental Law and Policy, Vol. 1, pp. 69-100, 1990. 
O tratamento diferenciado se refere a situações cujo princípio da igualdade soberana é deixado de lado para acomodar fatores, tais como diferentes níveis econômicos, de capacidade técnica, etc., estando profundamente ligado às reivindicações que fizeram surgir o direito internacional do desenvolvimento. Com efeito, o tratamento diferenciado no direito internacional tem como fundamento tanto a consideração de que as profundas desigualdades devem ser enfrentadas a fim de assegurar a legitimidade da própria ordem internacional quanto a própria necessidade de convergência de vários interesses em negociações internacionais. ${ }^{4}$

Do mesmo modo, os Estados são chamados a agir em colaboração para enfrentar os problemas ambientais globais. Contudo, há uma oposição entre a imperiosidade de uma resposta universal aos problemas ambientais globais e as necessidades dos países em desenvolvimento. O princípio das responsabilidades comuns mas diferenciadas e respectivas capacidades expressa essa oposição, mas ainda oferece margem para muitas discussões sobre base conceitual, assim como sobre seu próprio objetivo. ${ }^{5}$

O objetivo do princípio é a correção da desigualdade entre os Estados. No âmbito das negociações de acordos ambientais multilaterais, a desigualdade entre os Estados sempre aparece como um tema central de debate. ${ }^{6}$ A igualdade formal de participação no processo de negociação dos tratados não assegura que o seu resultado será justo. Enquanto há um consenso internacional do que sejam instituições e processos legítimos, a compreensão de justiça e de equidade no âmbito do direito internacional ainda é rudimentar. ${ }^{7}$

O tratamento diferenciado incorpora discriminações que objetivam fomentar a igualdade material entre os Estados. Normalmente, essa distinção se faz em termos de desenvolvimento. Procura-se incorporar tanto uma concepção de justiça corretiva - que tem como base corrigir as injustiças históricas - quanto distributiva - voltada para as desigualdades de hoje. ${ }^{8}$

Nesse sentido, o princípio tem como o foco a desigualdade entre países desenvolvidos e em desenvolvimento, que os coloca em posições muito distintas dentro do sistema internacional.

\footnotetext{
$4 \quad$ CULLET, P. Differential treatment in international law: towards a new paradigm of inter-state relations. European Journal Of International Law, [s.I], v. 10, n. 3, p.549-582, 1 mar. 1999. Oxford University Press (OUP). DOI: 10.1093/ejil/10.3.549.

$5 \quad$ FRENCH, Duncan. Developing States and International Environmental Law: The Importance of Differentiated Responsibilities. International \& Comparative Law Quarterly, [s.I], v. 49, n. 01, p.35-60, jan. 2000. Cambridge University Press (CUP). DOI: 10.1017/s0020589300063958.

6 O'NEIL, K. The Environment and International Relations. Cambridge: Cambridge University Press, 2009. aspects. Alphen aan den Rijn: Kluwer Law International, 2009.

8 CULLET, P. Differential treatment in international law: towards a new paradigm of inter-state relations. European Journal Of International Law, [s.I], v. 10, n. 3, p.549-582, 1 mar. 1999. Oxford University Press (OUP). DOI: 10.1093/ejil/10.3.549.
} 
O princípio foca, na maioria das vezes, na desigualdade entre os Estados em termos de desenvolvimento, o que os coloca em posições muito distintas no cenário internacional, demandando um tratamento diferenciado no âmbito da relação Norte-Sul. ${ }^{9}$ Estruturalmente, o tratamento diferenciado dos países em desenvolvimento objetiva não apenas reconhecer os limites da igualdade formal entre os Estados, mas também assegurar a redução dessas desigualdades, impedindo seu aumento e proporcionando resultados mais justos. ${ }^{10}$

Rajamani11 sintetiza duas justificativas para o tratamento diferenciado dos países em desenvolvimento no âmbito do direito internacional ambiental. A primeira, a autora chama de culpabilidade/titularidade e a segunda de consideração/capacidade.

\section{1 - O FUNDAMENTO DA CULPABILIDADE/TITULARIDADE OU RESPONSA- BILIDADE HISTÓRICA}

O fundamento da culpabilidade/titularidade, ou responsabilidade histórica, culpa os países desenvolvidos pela crise ambiental global. Por sua vez, como não contribuíram para tal crise e em virtude do passado de exploração colonial, os países em desenvolvimento devem gozar de compromissos menos rígidos, além de assistência técnica e financeira. ${ }^{12}$

French $^{13}$ identifica esse enfoque nos documentos pós-Brundtland e está especialmente insuflado nos documentos preparatórios da Rio-92. Considerando as diversas contribuições para a degradação do ambiente global, os Estados têm responsabilidades comuns, porém diferenciadas. De fato, os países desenvolvidos reconheceriam a responsabilidade que lhes cabe na busca internacional do desenvolvimento sustentável, tendo em vista as pressões exercidas por suas sociedades sobre o meio ambiente global e as tecnologias e os recursos financeiros que controlam.

Não é exatamente essa visão que Rajamani ${ }^{14}$ possui deste enfoque, quando afirma que é evidente que os países desenvolvidos, para atingir e manter o nível de desenvolvimento que possuem, pressionam muito o meio ambiente global. Diante das evidências que o meio ambiente não suporta tal pressão, os países desenvolvidos desejam impor a todos uma obrigação de mitigar suas externalidades ambientais. Os países em desenvolvimento são especialmente refratários a essa possibilidade por entenderem que ela procura, na verdade, manter o status quo do cenário

9 HONKONEN, Tuula. The common but differentiated responsibility principle in multilateral environmental agreements: regulatory and policy aspects. Alphen aan den Rijn: Kluwer Law International, 2009.

10 CULLET, P. Differential Treatment in Environmental Law: Addressing Critiques and Conceptualizing the Next Steps. Transnational Environmental Law, [s.I.], v. 5, n. 02, p.305-328, out. 2016. Cambridge University Press (CUP). Disponível em: http://dx.doi.org/10.1017/s204710251600025x. RAJAMANI, Lavanya. Differential Treatment in International Environmental Law. Oxford: Oxford University Press, 2006. RAJAMANI, Lavanya. Differential Treatment in International Environmental Law. Oxford: Oxford University Press, 2006.

FRENCH, Duncan. Developing States and International Environmental Law: The Importance of Differentiated Responsibilities. International \& Comparative Law Quarterly, [s.I], v. 49, n. 01, p.35-60, jan. 2000. Cambridge University Press (CUP). DOI: 10.1017/s0020589300063958.

RAJAMANI, Lavanya. Differential Treatment in International Environmental Law. Oxford: Oxford University Press, 2006. 


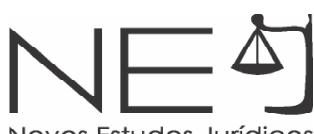

internacional, enfatizando que tais questões não podem ser tratadas sem considerar o passado de exploração colonial e a manutenção de uma estrutura internacional que os coloca em desvantagem.

Tratar-se-ia, bem salienta French ${ }^{15}$, de uma obrigação da comunidade internacional levar em conta as necessidades e as circunstâncias dos países em desenvolvimento quando implementam o direito internacional, cuja origem está nas históricas negociações econômicas que remontam à NOEl. Assim, partese do pressuposto que a proteção ambiental não seria uma prioridade para os países em desenvolvimento, que estão empenhados em garantir o crescimento econômico e a erradicação da pobreza.

Mesmo assim, o autor relata que o discurso de muitos países em desenvolvimento chama atenção que a responsabilidade dos países desenvolvidos vai além dos impactos negativos que suas atividades tiveram e têm no ambiente global, mas reforça que estes se beneficiaram economicamente de tal exploração, devendo contribuir de modo significativo para o enfrentamento.

A responsabilidade pela deterioração ambiental é mais visível quando se trata de poluição, ao mesmo tempo que a apropriação das riquezas geradas pela exploração dos recursos naturais está disponível apenas ao Norte. Porém, tanto Rajamani ${ }^{16}$ quanto French ${ }^{17}$ concordam que a afirmação de que o Norte é responsável pela degradação ambiental traz à tona um conjunto de questões intrincadas. A primeira delas é sobre a própria ligação dentre a responsabilidade ambiental e a contribuição para um determinado problema ambiental global. French ${ }^{18}$ argumenta que ainda que se concorde que a responsabilidade de um país deve ser proporcional à sua contribuição para um dano, em termos de degradação do meio ambiente global, a mensuração dessa responsabilidade encontra obstáculos técnicos e políticos.

Porém, para Rajamani ${ }^{19}$ tal discussão gira em torno do princípio do poluidor-pagador e das responsabilidades comuns mas diferenciadas ambos presentes na Declaração do Rio de 1992. O princípio do poluidor-pagador evidencia a ligação entre responsabilidade ambiental e contribuição para tal dano, sendo um corolário da noção do direito internacional costumeiro de que um Estado deve assegurar que as atividades sob sua jurisdição não causem dano a outros Estados. Essa obrigação, pondera French ${ }^{20}$, não admite nenhuma mitigação por considerações socioeconômicas, sendo igualmente exigida de todos os países.

\footnotetext{
$15 \quad$ FRENCH, Duncan. Developing States and International Environmental Law: The Importance of Differentiated Responsibilities. International \& Comparative Law Quarterly, [s.I], v. 49, n. 01, p.35-60, jan. 2000. Cambridge University Press (CUP). DOI: 10.1017/s0020589300063958. RAJAMANI, Lavanya. Differential Treatment in International Environmental Law. Oxford: Oxford University Press, 2006.

17 FRENCH, Duncan. Developing States and International Environmental Law: The Importance of Differentiated Responsibilities. International \& Comparative Law Quarterly, [s.I], v. 49, n. 01, p.35-60, jan. 2000. Cambridge University Press (CUP). DOI: 10.1017/s0020589300063958.

18 FRENCH, Duncan. Developing States and International Environmental Law: The Importance of Differentiated Responsibilities. International \& Comparative Law Quarterly, [s.I], v. 49, n. 01, p.35-60, jan. 2000. Cambridge University Press (CUP). DOI: 10.1017/s0020589300063958.

19 RAJAMANI, Lavanya. Differential Treatment in International Environmental Law. Oxford: Oxford University Press, 2006.

20 FRENCH, Duncan. Developing States and International Environmental Law: The Importance of Differentiated Responsibilities. International \& Comparative Law Quarterly, [s.I], v. 49, n. 01, p.35-60, jan. 2000. Cambridge University Press (CUP). DOI: 10.1017/s0020589300063958.
} 
No mesmo sentido, Honkonen ${ }^{21}$ salienta que o princípio das responsabilidades comuns mas diferenciadas chama todos os Estados à responsabilidade com o enfrentamento da degradação ambiental global, ao mesmo tempo que reconhece que os compromissos com esse enfrentamento devem ser distribuídos entre os Estados de acordo com diversas circunstâncias, entre as quais suas realidades socioeconômicas e, em especial, os imperativos de desenvolvimento.

A Declaração de Estocolmo sobre o Meio Ambiente Humano-72, especialmente considerando o contexto de participação dos países em desenvolvimento, trata da questão da diferenciação de modo muito cauteloso nos Princípios 11, 23 e 24. Vinte anos depois, foi a Rio-92 que representou uma afirmação veemente das responsabilidades diferenciadas entre os países desenvolvidos e em desenvolvimento na persecução da proteção ambiental global e promoção do desenvolvimento sustentável. Houve muito debate sobre o texto dos Princípios 6 e 7 que seriam inseridos na Declaração.

Cullet $^{22}$ assinala que o Princípio 7 da Declaração do Rio-92, que melhor articula o tratamento diferenciado opondo Norte e Sul, ainda é campo de divergências de interpretação. Os países do Norte, de modo especial os Estados Unidos, entendem que o mesmo não pode criar obrigações adicionais ao Norte, de modo que o princípio não pode impor obrigações hard law aos países desenvolvidos.

Rajamani ${ }^{23}$ evidencia a possibilidade de países desenvolvidos serem chamados a se responsabilizar com base em atividades potencialmente destrutivas. Trata-se, segundo French ${ }^{24}$, da instrumentalização dos conceitos de responsabilidade atual e responsabilidade conceitual.

Deste modo, alega-se que essa responsabilidade primária dos países desenvolvidos não deve ser presumida, mas compreendida por meio de uma distinção entre responsabilidade atual e responsabilidade conceitual. A responsabilidade atual relaciona-se ao estado da arte hoje, na qual os países desenvolvidos são os maiores contribuintes para a degradação ambiental global, sendo que a responsabilidade conceitual é um princípio geral que significa que a responsabilidade de cada país depende da sua contribuição para a degradação ambiental. A responsabilidade conceitual deixa aberta a possibilidade de que nem sempre serão os países desenvolvidos os responsáveis pela grande parte da degradação ambiental..$^{25}$

21 HONKONEN, Tuula. The common but differentiated responsibility principle in multilateral environmental agreements: regulatory and policy aspects. Alphen aan den Rijn: Kluwer Law International, 2009.

22 CULLET, P. Differential Treatment in Environmental Law: Addressing Critiques and Conceptualizing the Next Steps. Transnational Environmental Law, [s.I.], v. 5, n. 02, p.305-328, out. 2016. Cambridge University Press (CUP). Disponível em: http://dx.doi.org/10.1017/s204710251600025x. RAJAMANI, Lavanya. Differential Treatment in International Environmental Law. Oxford: Oxford University Press, 2006.

24 FRENCH, Duncan. Developing States and International Environmental Law: The Importance of Differentiated Responsibilities. International \& Comparative Law Quarterly, [s.I], v. 49, n. 01, p.35-60, jan. 2000. Cambridge University Press (CUP). DOI: 10.1017/s0020589300063958.

25 FRENCH, Duncan. Developing States and International Environmental Law: The Importance of Differentiated Responsibilities. International \& Comparative Law Quarterly, [s.I], v. 49, n. 01, p.35-60, jan. 2000. Cambridge University Press (CUP). DOI: 10.1017/s0020589300063958. 


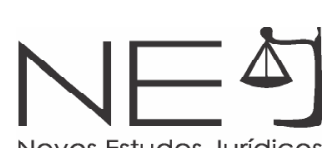

No mesmo sentido, Honkonen ${ }^{26}$ escreve que a dinâmica da realidade ao longo do tempo tende a modificar o modo de distribuição das obrigações ambientais internacionais hoje impostas principalmente aos países desenvolvidos. Deste modo, não seria razoável esperar que tais responsabilidades diferenciadas sempre serão impostas aos países desenvolvidos, deixando-se aberta a possibilidade de que sejam impostas aos países em desenvolvimento obrigações mais rígidas, na medida em que suas contribuições para a degradação ambiental aumentem.

Por sua vez, Rajamani²7 se opõe à possibilidade quando alega que isso não seria possível, uma vez que esses países são economias frágeis com sistemas políticos instáveis, cuja realidade socioeconômica pode mudar muito rapidamente e de modo drástico. Além disso, todo o esforço dos países em desenvolvimento é no sentido de evidenciar uma ligação direta entre a atividade e o dano, o que não seria o caso.

\section{2 - O FUNDAMENTO DA CONSIDERAÇÃO/CAPACIDADE DOS PAÍSES DESENVOLVIDOS}

Ajustificativa da consideração/capacidade alega que países com condições técnicas e financeiras melhores devem ajudar os demais na implementação de suas obrigações internacionais. Nesse sentido, os países desenvolvidos serão esses personagens com melhores condições/capacidades. Os efeitos humanos e ecológicos mais devastadores da degradação ambiental serão sentidos pelos países em desenvolvimento que não possuem estas capacidades. ${ }^{28}$

Honkonen ${ }^{29}$ também parece filiar-se a esta argumentação quando diz que a diferenciação de tratamento operacionalizada pelo princípio das responsabilidades comuns mas diferenciadas tem como fundamento as diferentes capacidades de reação dos Estados diante dos efeitos da degradação ambiental, assim como a capacidade de tomar medidas efetivas em nível interno.

Os países em desenvolvimento evidenciam que as obrigações de assistência técnica e/ou financeira não são uma caridade dos países desenvolvidos, uma vez que as estruturas institucionais para gerenciar essa assistência devem possuir uma adequada e equitativa representação do Norte e do Sul. ${ }^{30}$

26 HONKONEN, Tuula. The common but differentiated responsibility principle in multilateral environmental agreements: regulatory and policy aspects. Alphen aan den Rijn: Kluwer Law International, 2009.

27 RAJAMANI, Lavanya. Differential Treatment in International Environmental Law. Oxford: Oxford University Press, 2006.

28 RAJAMANI, Lavanya. Differential Treatment in International Environmental Law. Oxford: Oxford University Press, 2006; FRENCH, Duncan. Developing States and International Environmental Law: The Importance of Differentiated Responsibilities. International \& Comparative Law Quarterly, [s.I], v. 49, n. 01, p.35-60, jan. 2000. Cambridge University Press (CUP). DOI: 10.1017/s0020589300063958.

29 HONKONEN, Tuula. The common but differentiated responsibility principle in multilateral environmental agreements: regulatory and policy aspects. Alphen aan den Rijn: Kluwer Law International, 2009.

30 FRENCH, Duncan. Developing States and International Environmental Law: The Importance of Differentiated Responsibilities. International \& Comparative Law Quarterly, [s.I], v. 49, n. 01, p.35-60, jan. 2000. Cambridge University Press (CUP). DOI: 10.1017/s0020589300063958. 
Rajamani ${ }^{31}$ observa que os países do Norte preferem fundamentar sua liderança no fato de possuírem melhor capacidade técnica e financeira, em vez de contribuições históricas para o estado de degradação. Com isso, afasta-se a base jurídica do compromisso por um apelo moral, além de não reconhecer um direito ao desenvolvimento titularizado pelo Sul.

Os países desenvolvidos preferem ainda promover uma linguagem de 'parcerias', o que os países do Sul identificam como uma tentativa de diminuir ou mesmo esvaziar o princípio das responsabilidades comuns mas diferenciadas. Trata-se de uma argumentação que reacende a própria origem colonial do direito internacional, em que aqueles mais 'avançados' deveriam 'cuidar' dos atrasados, lembrando as palavras do artigo 22 do Tratado da Liga das Nações.

É interessante mencionar que, mesmo durante as negociações que levaram ao texto do Princípio 7 da Declaração do Rio, os Estados Unidos afirmaram que o compreendia no sentido de uma liderança dos países desenvolvidos no enfrentamento dos problemas ambientais devido ao seu status de poder econômico e experiência técnica, mas não no sentido do reconhecimento de obrigações internacionais ou mesmo de uma diminuição da responsabilidade dos países em desenvolvimento. ${ }^{32}$

Em síntese, Cullet ${ }^{33}$ ressalta que, no âmbito da governança global ambiental, o tratamento diferenciado também está ligado às responsabilidades diferenciadas sobre os causadores e sobre quem tem melhores condições técnicas e econômicas de enfrentá-lo. De fato, não se pode afirmar que todos os Estados possuem as mesmas responsabilidades em relação aos problemas ambientais globais, do mesmo modo que não possuem a mesma capacidade técnica.

O resultado final de toda essa contenda pode ser vislumbrado no texto do Princípio 7 da Declaração da Rio-92. No texto oficial, a responsabilidade dos países desenvolvidos projeta-se apenas para o futuro, enquanto a proposta do G77 deixa clara as responsabilidades que eles possuem, tendo em conta suas atividades que levaram ao atual estado de degradação ambiental.

O texto final também desagradou os países em desenvolvimento, que não puderam enxergar nele um meio de impor responsabilidade jurídica aos países desenvolvidos pelo estado atual da degradação ambiental. Além do mais, por meio do G77, esses países pleiteavam em sua proposta original uma obrigação de transferência de recursos financeiros e tecnológicos pelos países desenvolvidos como uma forma de compensação. Contudo, esse

31 RAJAMANI, Lavanya. Differential Treatment in International Environmental Law. Oxford: Oxford University Press, 2006.

32 FRENCH, Duncan. Developing States and International Environmental Law: The Importance of Differentiated Responsibilities. International \& Comparative Law Quarterly, [s.I], v. 49, n. 01, p.35-60, jan. 2000. Cambridge University Press (CUP). DOI: $10.1017 /$ s0020589300063958.

33 CULLET, P. Differential treatment in international law: towards a new paradigm of inter-state relations. European Journal Of International Law, [s.I], v. 10, n. 3, p.549-582, 1 mar. 1999. Oxford University Press (OUP). DOI: 10.1093/ejil/10.3.549. 


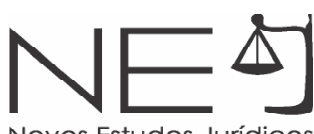

Novos Estudos Jurídicos

desejo não se refletiu no princípio. Quando a Declaração menciona a transferência de tecnologia e recursos, não o faz em tom impositivo. ${ }^{34}$

A desigualdade Norte-Sul refere-se a uma combinação de elementos de diversa natureza: econômica, social, ambiental, etc. Não se pode, de fato, negligenciar a justificativa histórica dessa desigualdade que ainda hoje permanece nos países em desenvolvimento, onde a pobreza é a principal causa de degradação ambiental e onde as deficiências econômicas e institucionais dos Estados não os faz suficientemente aptos para agir a contento. Em outras palavras, o tratamento diferenciado dos países em desenvolvimento justifica-se pela combinação da escassez de recursos para adimplir obrigações ambientais, o que os torna mais vulneráveis a desastres ambientais, além das desigualdades históricas e atuais. ${ }^{35}$

\section{3 - NORMAS DIFERENCIAIS, NORMAS CONTEXTUAIS E NORMAS ABSOLUTAS: INSTRUMENTALIZANDO A DIFERENCIAÇÃO}

Sobre a aplicação normativa do tratamento diferenciado dos países em desenvolvimento, Stone $^{36}$ afirma que todos os acordos internacionais possuem um impacto diferenciado em cada Parte. Contudo, a noção de responsabilidades comuns mas diferenciadas refere-se exclusivamente àquela diferenciação que se faz no próprio texto do acordo. Essa diferenciação pode se operar por meio de obrigações substantivas, de cronogramas de implementação mais favoráveis, permissão de uso de determinadas excludentes, ser mais leniente com situações de incumprimento, imposição de obrigações de assistência técnica e financeira a determinados grupos de Estados, etc.

Magraw $^{37}$ observa a existência de três tipos de normas internacionais que também se fazem presente no direito internacional ambiental e no modo como este instrumentaliza o tratamento diferenciado: normas diferenciais, normas contextuais e normas absolutas. O primeiro tipo de norma são as normas diferenciais, entendidas como aquelas normas que fazem uma diferenciação explícita entre determinados grupos de Estados. Essa distinção pode ser feita em várias bases, inclusive com base no estágio de desenvolvimento, levando em conta mais de um interesse. Rajamani ${ }^{38}$ (2006), também com base no mesmo autor, prefere chamar essas normas diferenciais de normas diferenciais explícitas.

\footnotetext{
$34 \quad$ FRENCH, Duncan. Developing States and International Environmental Law: The Importance of Differentiated Responsibilities. International \& Comparative Law Quarterly, [s.I], v. 49, n. 01, p.35-60, jan. 2000. Cambridge University Press (CUP). DOI: 10.1017/s0020589300063958.

35 HONKONEN, Tuula. The common but differentiated responsibility principle in multilateral environmental agreements: regulatory and policy aspects. Alphen aan den Rijn: Kluwer Law International, 2009.

36 STONE, Christopher D. Common but Differentiated Responsibilities in International Law. The American Journal Of International Law, [s.I], v. 98 , n. 2, p.276-301, abr. 2004. JSTOR. DOI: 10.2307/3176729.

37 MAGRAW, Daniel Barstow. Legal treatment of developing countries. Colorado Journal of International Environmental Law and Policy, Vol. 1, pp. 69-100, 1990.

38 RAJAMANI, Lavanya. Differential Treatment in International Environmental Law. Oxford: Oxford University Press, 2006.
} 
Por sua vez, segundo Magraw ${ }^{39}$, as normas contextuais conferem o mesmo tratamento a todos os Estados, mas permitem que a implementação seja feita levando em conta características que podem variar entre os Estados, o que implica em uma consideração de vários interesses. A indeterminação aparece como uma característica destas normas, o que confere aos Estados amplas margens para implementação. Essa indeterminação pode estar presente em normas contextuais gerais - que não limitam as circunstâncias que podem ser invocadas - e aquelas consideradas como limitadas e, tão logo, possuem uma delimitação estrita das condições e das circunstâncias que podem ser invocadas.

Normas contextuais parecem ser universalmente aplicáveis, mas requerem ou permitem levar em conta outros fatores na sua aplicação, especialmente de caráter socioeconômico. Expressões como 'razoável' ou 'equitativo' indicam este tipo de norma. A elasticidade dessas normas não pode ser infinita e, nesse sentido, os próprios tratados procuram limitá-las. Contudo, essas normas possuem um papel vital no direito internacional exatamente pela sua flexibilidade. ${ }^{40}$

Como uma terceira categoria de normas internacionais, Magraw ${ }^{41}$ refere-se às normas absolutas, entendidas como aquelas que estabelecem a mesma obrigação a todos os Estados e não prevê - ou até mesmo não permite - que sejam feitas considerações outras - tais como considerações socioeconômicas - no sentido de permitir uma flexibilização de sua aplicação.

Em diálogo com classificação de Magraw $^{42}$, Rajamani ${ }^{43}$ provê um panorama mais abrangente e divide as normas de tratamento diferenciado em três categorias, em vez de duas, ao considerar também as normas de assistência. São elas: diferenciação em termos de obrigações centrais dos tratados, diferenciação em termos de implementação e diferenciação em termos de obrigações de assistência financeira e tecnológica.

As primeiras obrigações referem-se a uma diferenciação entre os Estados no que toca às obrigações fundamentais do tratado, ou seja, aquelas que instrumentalizam a realização do seu objetivo. Essas regras podem aparecer tanto em tratados multilaterais quanto em tratados regionais que lidem com questões ambientais transfronteiriças. ${ }^{44}$

\footnotetext{
39 MAGRAW, Daniel Barstow. Legal treatment of developing countries. Colorado Journal of International Environmental Law and Policy, Vol. 1, pp. 69-100, 1990.

40 FRENCH, Duncan. Developing States and International Environmental Law: The Importance of Differentiated Responsibilities. International \& Comparative Law Quarterly, [s.I], v. 49, n. 01, p.35-60, jan. 2000. Cambridge University Press (CUP). DOI: 10.1017/s0020589300063958.

41 MAGRAW, Daniel Barstow. Legal treatment of developing countries. Colorado Journal of International Environmental Law and Policy, Vol. 1, pp. 69-100, 1990.

42 MAGRAW, 1990

43 RAJAMANI, Lavanya. Differential Treatment in International Environmental Law. Oxford: Oxford University Press, 2006.

44 RAJAMANI, Lavanya. Differential Treatment in International Environmental Law. Oxford: Oxford University Press, 2006.
} 
Por sua vez, Rajamani ${ }^{45}$ esclarece que, tendo em vista as necessidades técnicas dos países em desenvolvimento e as dificuldades que podem enfrentar na implementação dos tratados ambientais, os mesmos têm por prática a incorporação de normas que visam facilitar tal processo. Essas normas, cuja diferenciação ocorre em nível de implementação, podem ser divididas em normas que conferem um contexto diferente para a implementação, normas que prorrogam os prazos de execução, permissão para adotar anos-base subsequentes, mecanismos de monitoramento mais flexíveis e mesmo enfoques mais leves para situações de inadimplemento.

Além disso, a diferenciação também aparece por meio da garantia de assistência técnica e financeira aos países em desenvolvimento. Rajamani ${ }^{46}$ considera essas normas como uma categoria de diferenciação que se materializa por meio de assistência financeira, intercâmbio de informações, transferência de tecnologia e mesmo treinamento para o desenvolvimento de capacidades.

\section{2 - AS CONTRIBUIÇÕES NACIONALMENTE DETERMINADAS NO ACORDO DE PARIS: TRATAMENTO DIFERENCIADO PARA TODOS?}

O Acordo de Paris, resultado direto da decisão da Conferência das Partes (COP) da CQMC em Durban, concluído na COP21-Paris, em 12 de dezembro de 2015, está assinado por 191 países, todos Partes da CQMC, tendo sido ratificado por 129 Partes, e entrou em vigor no dia 4 de novembro de 2016. Ele está estruturado em 29 artigos - apenas um a mais do que o Protocolo de Quioto ${ }^{47}$, seu antecessor. Ele não contém anexos e mesmo os seus artigos não são nomeados.

A CQMC é, sem dúvida, muito favorável aos países em desenvolvimento, ao ponto de Roberts e Parks ${ }^{48}$ afirmarem que ela é o documento que melhor reflete concepções de justiça climática do modo por eles entendida. De fato, Rajamani ${ }^{49}$ relata que a relação entre o Acordo e a Convenção foi objeto de disputa entre os países desenvolvidos e em desenvolvimento durante todo o processo de negociação. Os primeiros entendiam que o Acordo deveria ser visto como uma mudança de paradigma em relação à Convenção, enquanto os últimos insistiam que o Acordo deriva da Convenção e, portanto, deve ser interpretado e implementado à luz dela. O caput do artigo 2 do Acordo demarca uma conciliação entre essas posições quando afirma que o Acordo reforça a implementação da Convenção, incluindo seu objetivo.

\footnotetext{
$45 \quad$ RAJAMANI, Lavanya. Differential Treatment in International Environmental Law. Oxford: Oxford University Press, 2006.

46 RAJAMANI, Lavanya. Differential Treatment in International Environmental Law. Oxford: Oxford University Press, 2006.

47 A seguir referido apenas como 'Protocolo'.

48 PARKS, Bradley C; ROBERTS, J Timmons. Inequality and the global climate regime: breaking the north-south impasse. Cambridge Review Of International Affairs, [s.I.], v. 21, n. 4, p.621-648, dez. 2008. Informa UK Limited. Disponível em: http://dx.doi.org/10.1080/ 09557570802452979.

49 RAJAMANI, Lavanya. Ambition And Differentiation In The 2015 Paris Agreement: Interpretative Possibilities And Underlying Politics. International And Comparative Law Quarterly, [s.I.], v. 65, n. 02, p.493-514, 16 mar. 2016. Cambridge University Press (CUP). Disponível em: http://dx.doi. org/10.1017/s0020589316000130.
} 


\section{1 - AS CONTRIBUIÇÕES NACIONALMENTE DETERMINADAS (CND) NO ACORDO DE PARIS}

Os primeiros artigos do Acordo não trazem nada de novo, em se considerando sua localização cartográfica e a prática dos tratados. O artigo primeiro traz definições, utilizando-se das mesmas endossadas no texto convencional. Por sua vez, o artigo segundo trata exatamente dos objetivos do Acordo ou, por assim dizer, da medida de sua ambição como resposta às mudanças climáticas.

Em termos climáticos, o artigo 2.1(a) traça como objetivo, a fim de reduzir significativamente os riscos e os impactos da mudança climática, manter o aumento da temperatura média global bem abaixo de $2^{\circ} \mathrm{C}$ em relação aos níveis pré-industriais, e envidar esforços para limitar esse aumento da temperatura a $1,5^{\circ} \mathrm{C}$ em relação aos níveis pré-industriais. Para tanto, o artigo 4.2 traz aquela que é a principal inovação do Acordo de Paris em relação a Quioto, quando estabelece que todas as Partes devem (shall, na versão inglesa) perseguir medidas domésticas de mitigação, de acordo com contribuições nacionalmente determinadas (CND) $)^{50}$ que devem preparar, comunicar e manter.

A princípio, tal obrigação não se constitui em algo necessariamente novo, uma vez que a própria CQMC, no seu artigo 4.1 (a) e (b), estabelece que todas as Partes devem elaborar, atualizar periodicamente e publicar inventários nacionais de emissões antrópicas e das remoções por sumidouros, assim como formular, implementar, publicar e atualizar políticas nacionais e/ou regionais que incluam medidas de mitigação e adaptação. Porém, é por meio dessas contribuições que o Acordo pretende operacionalizar o tratamento diferenciado. A natureza jurídica das contribuições nacionalmente determinadas foi debatida durante os processos de negociação. Bodansky ${ }^{51}$ ressalta que a União Europeia, a fim de dar maior credibilidade, assim como assegurar a implementação e a observância, desejava estabelecer que as Partes tivessem a obrigação de implementar/atingir suas contribuições. Por sua vez, os Estados Unidos foram contra essa proposta, alegando que um sistema de transparência cumpriria os mesmos objetivos.

Três itens do artigo quarto (4.2, 4.3 e 4.4) merecem uma análise destacada, uma vez que sepultam definitivamente a lógica endossada em Quioto e dão base para uma nova arquitetura do regime de mudanças climáticas no âmbito da CQMC, traçando um novo perfil para a base de diferenciação entre os países.

O princípio das responsabilidades comuns mas diferenciadas e respectivas capacidades é, de

50 A Decisão n' 1/CP19, na COP de Warsaw em 2013, já convidou as Partes a submeter o que denominou de 'intended nationally determined contributions'.

51 BODANSKY, Daniel. The Paris Climate Change Agreement: A New Hope?. The American Journal Of International Law, [s.I.], v. 110, n. 2, p.288319, 2016. American Society of International Law. Disponível em: http://dx.doi.org/10.5305/amerjintelaw.110.2.0288. 


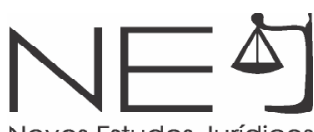

fato, o elemento estrutural da CQMC e deve ser de todo o sistema jurídico constituído a partir dela, assim como o Protocolo de Quioto o instrumentalizou seguindo a lógica binária entre países desenvolvidos e países em desenvolvimento. Porém, essa diferenciação é um tema de enorme disputa no âmbito das negociações que se seguiram ao Protocolo, sendo que o Acordo de Copenhague, a despeito de sua ausência de status jurídico, representou uma inflexão no rumo do tratamento diferenciado nesta seara do direito internacional.

De plano, deve-se analisar algo que salta aos olhos diante do texto do Acordo de Paris. Ele menciona o princípio das responsabilidades comuns mas diferenciadas e respectivas capacidades quatro vezes: no preâmbulo, no artigo 2.2 e nos artigos 4.3 e 4.19. Nessas menções ele traz um novo elemento ao princípio quando menciona a diferenciação 'à luz das diferentes circunstâncias nacionais'.

Por sua vez, a chave para compreender o impacto de tais contribuições nacionalmente determinadas - em especial aquelas que emanarão dos países desenvolvidos - é prestar atenção nos artigos 4.3 e 4.4 do Acordo. Utilizando-se de uma linguagem imprecisa - uma das características da soft law -, determina-se que tais contribuições representarão (will, na versão inglesa) uma progressão e as mais altas ambições, refletindo as responsabilidades comuns mas diferenciadas e respectivas capacidades, à luz das diferentes circunstâncias nacionais. Sobre a ambição progressiva das contribuições nacionalmente determinadas, Rajamani ${ }^{52}$ obtempera que o artigo 4.3 estabelece uma forte expectativa, mas não uma obrigação para as Partes. Além disso, de acordo com a própria natureza das contribuições nacionalmente determinadas, é a própria Parte que determina se sua nova contribuição preenche tal requisito.

Nessa esteira, se estabelece que os países desenvolvidos devem (should, na versão inglesa) continuar tomando a liderança por meio de metas de redução de emissão absoluta na economia de modo abrangente, enquanto os países em desenvolvimento devem continuar envidando esforços de mitigação e, ao longo do tempo, também adotar metas de redução à luz de suas circunstâncias nacionais.

\section{2 - PERSPECTIVAS DO TRATAMENTO DIFERENCIADO DOS PAÍSES EM DE- SENVOLVIMENTO A PARTIR DO ACORDO DE PARIS}

É interessante notar que a base da diferenciação no âmbito do direito internacional ambiental encerra uma contenda sobre sua justificativa com base na responsabilidade histórica dos países desenvolvidos ou com base na capacidade técnica e financeira que eles têm para o adequado enfrentamento dos problemas ambientais. Em virtude dessa disputa, desde a Declaração do

52 RAJAMANI, Lavanya. Ambition And Differentiation In The 2015 Paris Agreement: Interpretative Possibilities And Underlying Politics. International And Comparative Law Quarterly, [s.l.], v. 65, n. 02, p.493-514, 16 mar. 2016. Cambridge University Press (CUP). Disponível em: http://dx.doi. org/10.1017/s0020589316000130. 
Rio-92, e mesmo nos textos da CQMC e do Protocolo, esse tratamento aparece como princípio das responsabilidades comuns mas diferenciadas e respectivas capacidades, mas o Acordo inova ao condicionar a observação do princípio também às diferentes circunstâncias nacionais.

De fato, a primeira vez que o princípio aparece condicionado desta forma foi na Declaração Presidencial Conjunta sobre Mudanças Climáticas entre os Estados Unidos e a China em novembro de 2014, exatamente tendo em vista a COP de Paris. Do ponto de vista jurídico, Maljean-Dubois ${ }^{53}$ chama atenção que essa condicionante atende principalmente o desejo dos países desenvolvidos de poderem alegar um leque maior de fatores para diferenciação, promovendo o que entendem por ser uma interpretação evolutiva do princípio. O princípio das responsabilidades comuns mas diferenciadas, ao materializar o tratamento diferenciado dos países em desenvolvimento no âmbito da política ambiental, é reflexo direito do que McGee e Steffek ${ }^{54}$ chamam de multilateralismo redistributivo, cujas origens estão na NOEI e na resistência pós-descolonização. Maljean-Dubois ${ }^{55}$ observa, porém, que depois de 1997 o mundo teria se tornado bem mais complexo, com a evidência de grandes emissores também nos países do Sul, mas ainda albergados sob o rótulo de países em desenvolvimento. Ela também salienta que as alianças também se tornaram mais complexas, observando-se acordos e estratégias entabuladas para além da dicotomia Norte-Sul. Depois de Copenhague ficou clara a intenção de transformar o modelo proposto em Quioto, tendo em vista exatamente uma reação ao multilateralismo distributivo.

Sobre os argumentos de desmantelar o tratamento diferenciado com base na ascensão de países como a China e mesmo em uma melhora da situação dos países do Sul, Cullet ${ }^{56}$ afirma que, em que pese a melhora de alguns países, não houve nenhuma mudança estrutural significante nos últimos anos que justifique o abandono da diferenciação. De fato, o tratamento diferenciado deve ser interpretado de forma dinâmica, desde que as desigualdades estruturais demonstrem sinais fortes de diminuição.

De fato, o Acordo de Paris representa uma ruptura quando assinala a primazia da política doméstica sobre as negociações internacionais. O Acordo, na verdade, põe de lado os conflitos distributivos que marcaram as negociações climáticas pós-Quioto ao reconhecer que os grandes emissores simplesmente não poderiam ser forçados a adimplir cortes drásticos de emissões.

53 MALJEAN-DUBOIS, Sandrine. The Paris Agreement: A New Step in the Gradual Evolution of Differential Treatment in the Climate Regime?. Review Of European, Comparative \& International Environmental Law, [s.l.], v. 25, n. 2, p.151-160, jul. 2016. Wiley-Blackwell. Disponivel em: http://dx.doi.org/10.1111/reel.12162.

54 MCGEE, Jeffrey; STEFFEK, Jens. The Copenhagen Turn in Global Climate Governance and the Contentious History of Differentiation. Journal Of Environmental Law, [s.l.], v. 28, n. 1, p.37-63, mar. 2016. Oxford University Press (OUP). Disponível em: http://dx.doi.org/10.1093/jel/ eqw003.

55 MALJEAN-DUBOIS, Sandrine. The Paris Agreement: A New Step in the Gradual Evolution of Differential Treatment in the Climate Regime?. Review Of European, Comparative \& International Environmental Law, [s.l.], v. 25, n. 2, p.151-160, jul. 2016. Wiley-Blackwell. Disponivel em: http://dx.doi.org/10.1111/reel.12162.

56 CULLET, P. Differential Treatment in Environmental Law: Addressing Critiques and Conceptualizing the Next Steps. Transnational Environmenta Law, [s.I.], v. 5, n. 02, p.305-328, out. 2016. Cambridge University Press (CUP). Disponível em: http://dx.doi.org/10.1017/s204710251600025x. 
Ao mesmo tempo em que salienta que o Acordo de Paris inaugura uma nova lógica na política internacional do clima, Falkner ${ }^{57}$ assinala que o mesmo adota um enfoque mais realístico e apto a assegurar uma cooperação duradoura.

Trata-se de uma estratégia de autodiferenciação que resulta de um processo voluntário de autodeterminação de suas contribuições, de modo a colocar de lado o conflito Norte-Sul e permitir uma maior diferenciação, abandonando o que se entende por uma divisão estática dos anexos do Protocolo de Quioto. Observe-se que o Acordo não prevê nada sobre a natureza ou mesmo as ambições das contribuições nacionalmente determinadas. ${ }^{58}$

É fato que o Acordo de Paris resulta do compromisso firmado entre os maiores emissores países em desenvolvimento e os Estados Unidos. Mesmo não sendo mais o maior emissor de gases de efeito

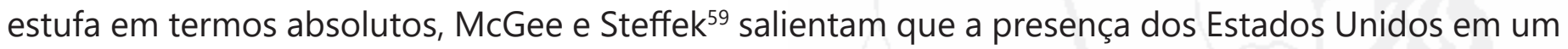
acordo climático é essencial para sua efetividade. Porém, a resistência que aquele país demonstra ao multilateralismo redistributivo - cujo tratamento diferenciado dos países em desenvolvimento é uma das consequências - já se fazia sensível desde mesmo a Conferência da Rio-92 e no âmbito da governança global do comércio, desde as negociações que levaram à Conferências das Nações Unidas sobre Comércio e Desenvolvimento.

Pauwelyn ${ }^{60}$ observa que o tratamento diferenciado da forma como foi emplacado pelos países em desenvolvimento, sobretudo pela ação de movimentos políticos e coalizações ao longo dos anos 70, realmente está fadado ao desparecimento, ou seja, a ideia de uma divisão rígida entre países desenvolvidos e em desenvolvimento. Em seu lugar surge uma nova abordagem da diferenciação que leva em conta todo um conjunto de variáveis que tal divisão estática não abarca. 0 tratamento diferenciado dos países em desenvolvimento, materializado como uma exceção ao princípio da reciprocidade, adentrou o GATT por meio de uma decisão em 1979. Porém, os Acordos da OMC, concluídos em 1994, terminaram esvaziando o princípio de não reciprocidade em termos de obrigações centrais.

57 FALKNER, Robert. The Paris Agreement and the new logic of international climate politics. International Affairs, [s.I.], v. 92, n. 5, p.1107-1125, 31 ago. 2016. Oxford University Press (OUP). Disponível em: http://dx.doi.org/10.1111/1468-2346.12708.

58 MALJEAN-DUBOIS, Sandrine. The Paris Agreement: A New Step in the Gradual Evolution of Differential Treatment in the Climate Regime?. Review Of European, Comparative \& International Environmental Law, [s.l.], v. 25, n. 2, p.151-160, jul. 2016. Wiley-Blackwell. Disponível em: http://dx.doi.org/10.1111/reel.12162.

59 MCGEE, Jeffrey; STEFFEK, Jens. The Copenhagen Turn in Global Climate Governance and the Contentious History of Differentiation. Journal Of Environmental Law, [s.I.], v. 28, n. 1, p.37-63, mar. 2016. Oxford University Press (OUP). Disponível em: http://dx.doi.org/10.1093/jel/ eqw003.

60 PAUWELYN, Joost. The End of Differential Treatment for Developing Countries? Lessons from the Trade and Climate Change Regimes. Rev Euro Comp \& Int Env Law, [s.I], v. 22, n. 1, p.29-41, abr. 2013. Wiley-Blackwell. DOI: 10.1111/reel.12017. 
O Acordo de Paris, como observam McGee e Steffek ${ }^{61}$, é o resultado de um movimento iniciado pelos Estados Unidos - juntamente com outros países desenvolvidos - em Copenhague em direção a outra interpretação do princípio das responsabilidades comuns mas diferenciadas. Deixa-se de lado uma diferenciação com base em compromissos prescritivos, internacionalmente negociados, para um modelo de diferenciação para todos, com base em uma noção subjetiva de 'diferentes circunstâncias nacionais'. O Acordo de Paris, sob a promessa de uma maior diferenciação, na verdade viabiliza uma diferenciação fraca entre os países, afastando-se totalmente noções de equidade e permitindo-os apenas uma diferenciação que fazem por si mesmos.

Falkner ${ }^{62}$ é enfático quando assinala constantemente que o Acordo de Paris põe de lado os conflitos distributivos que marcam a CQMC e que estiveram particularmente presentes em Quioto. Criticando a abordagem do Protocolo, o autor mesmo afirma que o potencial conflitivo que ele carregava foi o principal obstáculo para que o modelo se mantivesse. Para McGee e Steffek ${ }^{63}$, partindo de uma perspectiva histórica da atuação dos Estados Unidos, isso representa um forte movimento contra a agenda intervencionista e redistributiva de Quioto, algo que já ocorreu em tantas outras searas da governança global.

Porém, é interessante notar que, mesmo em um tom entusiasta com o Acordo de Paris por ter deixado de lado o conflito Norte-Sul, Falkner ${ }^{64}$ é cético quando se questiona se o mesmo, do modo como está redigido, poderá ser efetivo no propósito fixado no artigo 2.1. Para tanto, deve-se observar o cuidado com que as obrigações no artigo 4 foram redigidas. Ao apelar para as contribuições nacionalmente determinadas, o artigo 4 não contém nenhuma obrigação substantiva, desdobrando-se em obrigações procedimentais sobre como essas contribuições devem ser comunicadas no âmbito da CQMC. Rajamani ${ }^{65}$ pondera que as Partes estão sujeitas a obrigações vinculantes de conduta em relação às contribuições nacionalmente determinadas. Há apenas uma expectativa de boa-fé que as Partes atingirão suas metas por meio das ações que implementarão, apenas. Deste modo, as contribuições nacionalmente determinadas não são executáveis, nem no plano internacional nem no doméstico.

$61 \quad$ MCGEE, Jeffrey; STEFFEK, Jens. The Copenhagen Turn in Global Climate Governance and the Contentious History of Differentiation. Journa Of Environmental Law, [s.I.], v. 28, n. 1, p.37-63, mar. 2016. Oxford University Press (OUP). Disponível em: http://dx.doi.org/10.1093/jel/ eqw003.

62 FALKNER, Robert. The Paris Agreement and the new logic of international climate politics. International Affairs, [s.I.], v. 92, n. 5, p.1107-1125, 31 ago. 2016. Oxford University Press (OUP). Disponível em: http://dx.doi.org/10.1111/1468-2346.12708.

63 MCGEE, Jeffrey; STEFFEK, Jens. The Copenhagen Turn in Global Climate Governance and the Contentious History of Differentiation. Journa Of Environmental Law, [s.l.], v. 28, n. 1, p.37-63, mar. 2016. Oxford University Press (OUP). Disponível em: http://dx.doi.org/10.1093/jel/ eqw003.

64 FALKNER, Robert. The Paris Agreement and the new logic of international climate politics. International Affairs, [s.l.], v. 92, n. 5, p.1107-1125, 31 ago. 2016. Oxford University Press (OUP). Disponível em: http://dx.doi.org/10.1111/1468-2346.12708.

65 RAJAMANI, Lavanya. Ambition And Differentiation In The 2015 Paris Agreement: Interpretative Possibilities And Underlying Politics. Internationa And Comparative Law Quarterly, [s.I.], v. 65, n. 02, p.493-514, 16 mar. 2016. Cambridge University Press (CUP). Disponível em: http://dx.doi. org/10.1017/s0020589316000130. 
O artigo 4 do Acordo de Paris representa uma espécie de virada normativa no âmbito do tratamento diferenciado dos países em desenvolvimento. Até então, a CQMC e o seu Protocolo sempre foram tidos como a aplicação mais perfeita do tratamento diferenciado dos países em desenvolvimento, operacionalizado por meio de normas diferenciais ${ }^{66}$, também chamadas de normas de diferenciação em relação às obrigações centrais do tratado ${ }^{67}$. Porém, a diferenciação que o artigo 4 operacionaliza, festejada como uma 'diferenciação para todos', ocorre por meio do que Magraw $^{68}$ chama normas contextuais, entendendo-se aqueles que, a princípio, provêm o mesmo tratamento a todos os países, mas possuem condicionantes que permitem uma diferenciação na sua implementação, no caso, essa diferenciação ocorre por meio das 'circunstâncias nacionais'.

Maljean-Dubois ${ }^{69}$ atenta para o que se entende ser a maior fraqueza do modelo de contribuições nacionalmente determinadas endossado pelo Acordo. De fato, tal modelo delega aos países muita flexibilidade na determinação de suas contribuições nacionais. Magraw ${ }^{70}$ afirma que as normas contextuais, marcadas pela indeterminação, possibilitam um amplo leque para argumentar observância/inobservância e mesmo se fossem judicializadas conduziriam a um resultado incerto. Deste modo, os países em desenvolvimento devem apresentar contribuições nacionais que levem em conta suas necessidades específicas e circunstâncias especiais (art.3.2 da CQMC) e mesmo o direito que possuem ao desenvolvimento sustentável (art.3.4 da CQMC) e crescimento econômico (art. 3.5 da CQMC).

Magraw ${ }^{71}$ levanta quatro cenários ideais para o uso de normas de diferenciação contextual: a) facilitação do acordo, ao mesmo em que reconhece a legitimidade de determinadas preocupações para o conjunto dos Estados; b) problemas marcados pela incerteza, seja científica ou relacionada às situações fáticas a serem cobertas, permitindo uma flexibilidade para que o nível ótimo de resposta evolua com o tempo; c) situações em que os custos de elaborar normas precisas determinando todos os meandros possíveis seria demasiadamente alto, tendo em vista os benefícios advindos dela; e d) situações em que é claro o que se deve fazer, mas existe a possibilidade de mudanças de cenário que podem afetar o modo de enfrentamento.

\footnotetext{
$66 \quad$ MAGRAW, Daniel Barstow. Legal treatment of developing countries. Colorado Journal of International Environmental Law and Policy, Vol. 1, pp. 69-100, 1990.

67 RAJAMANI, Lavanya. Differential Treatment in International Environmental Law. Oxford: Oxford University Press, 2006.

68 MAGRAW, Daniel Barstow. Legal treatment of developing countries. Colorado Journal of International Environmental Law and Policy, Vol. 1, pp. 69-100, 1990.

69 MALJEAN-DUBOIS, Sandrine. The Paris Agreement: A New Step in the Gradual Evolution of Differential Treatment in the Climate Regime?. Review Of European, Comparative \& International Environmental Law, [s.I.], v. 25, n. 2, p.151-160, jul. 2016. Wiley-Blackwell. Disponível em: http://dx.doi.org/10.1111/reel.12162.

70 MAGRAW, Daniel Barstow. Legal treatment of developing countries. Colorado Journal of International Environmental Law and Policy, Vol. 1, pp. 69-100, 1990.

71 MAGRAW, Daniel Barstow. Legal treatment of developing countries. Colorado Journal of International Environmental Law and Policy, Vol. 1, pp. 69-100, 1990.
} 
Deste modo, as normas contextuais podem dar a falsa impressão de progresso, quando ocultam a indisposição dos Estados. As normas diferenciais no Acordo de Paris, ao deixarem de lado o nível de desenvolvimento como valor considerado para modular as CND, optam por uma condicionalidade impassível de qualquer escrutínio internacional. A redução das emissões e a transição para uma economia de baixo carbono são imperativas aos Estados que ainda resistem por motivos variados, que vão desde uma incapacidade de acompanhar o estado das tecnologias limpas - e com isso perdendo posições no mercado mundial - ou mesmo pela pressão de setores internos da economia e extremamente poluentes.

Sob a promessa de maior diferenciação, esse modelo endossado pelo Acordo de Paris coloca riscos tais como um nivelamento por baixo, que somente pode ser evitado por critérios objetivos combinados com um mínimo de controle e proteção multilateral, além de enfraquecer o poder de barganha dos países em desenvolvimento como grupo e mesmo levantar incertezas sobre a própria operacionalização desse modo contextual de diferenciação. ${ }^{72}$

Magraw $^{73}$ afirma que mesmo normas contextuais, como a do Acordo sobre as contribuições nacionalmente determinadas, podem ser gerais ou limitadas, quando atêm claramente as situações que podem ser levadas em conta no âmbito de aplicação daquela norma. Todavia, Maljean-Dubois ${ }^{74}$ salienta que o Acordo de Paris, ao mesmo tempo em que favoreceu uma abordagem descendente, esvaziou elementos impositivos para garantir sua implementação e um nível crescente de ambição.

O Acordo de Paris - cujo embrião já estava em Copenhague - marca a vitória da estratégia revisionista endossada pelo Norte, principalmente pelos Estados Unidos. Deste modo, de acordo com Kiely ${ }^{75}$, não se deve crer que a hegemonia dos EUA não esteja sendo desafiada em vários setores da política internacional, principalmente por países do Sul, mais notadamente os emergentes. Porém, deve-se ter em conta o papel preponderante dos EUA como líder da globalização econômica. O ponto é que esse desafio, em termos econômicos, tem retratado com exagero. Os Estados Unidos até passam por dificuldades, podendo-se até mesmo questionar sua capacidade de lidar adequadamente com os ainda persistentes efeitos da crise financeira de 2008. Afirmar um declínio dos Estados Unidos tendo em vista sua questionável capacidade de enfrentar a crise é diferente de justificar tal declínio, devido à emergência de um país, notadamente a China, ou conjunto de países do Sul - como os

72 PAUWELYN, Joost. The End of Differential Treatment for Developing Countries? Lessons from the Trade and Climate Change Regimes. Rev Euro Comp \& Int Env Law, [s.I], v. 22, n. 1, p.29-41, abr. 2013. Wiley-Blackwell. DOI: 10.1111/reel.12017.

73 MAGRAW, Daniel Barstow. Legal treatment of developing countries. Colorado Journal of International Environmental Law and Policy, Vol. 1, pp. 69-100, 1990.

74 MALJEAN-DUBOIS, Sandrine. The Paris Agreement: A New Step in the Gradual Evolution of Differential Treatment in the Climate Regime?. Review Of European, Comparative \& International Environmental Law, [s.l.], v. 25, n. 2, p.151-160, jul. 2016. Wiley-Blackwell. Disponivel em: http://dx.doi.org/10.1111/reel.12162.

75 KIELY, Ray. The rise and fall of emerging powers: Globalisation, US Power and the Global North-South divide. Londres: Palgrave Macmillian, 2016. (Global Reordering). 


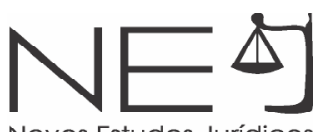

BASIC - uma vez que, para a eles, a crise também é um problema até maior do que é para os Estados Unidos. Vitória da estratégia dos Estados Unidos que, pouco depois, abandona o Acordo. Por meio de uma interpretação tendo em conta os cânones estabelecidos pela Convenção de Viena sobre o Direito dos Tratados e mesmo a intrincada arquitetura do Acordo de Paris ao combinar obrigações de diversos graus de precisão, vinculatividade e obrigação, Rajamani e Brunnée ${ }^{76}$, analisando a saída dos Estados Unidos do Acordo de Paris, sustentam que um país não pode, sem com isso ferir o direito internacional, diminuir suas contribuições nacionalmente determinadas ou mesmo retrocedê-las, sob pena de frustrar o objetivo do tratado.

O Acordo de Paris, quando escamoteia com o dilema Norte-Sul, de acordo com Cullet ${ }^{77}$, não representa um modelo para o futuro, mas apenas uma vitória diante do nada, ou seja, ele é apenas melhor do que acordo nenhum. Crítico ao modo como a diferenciação foi operacionalizada no Acordo de Paris, o autor vê as contribuições nacionalmente determinadas à luz das circunstâncias nacionais como autodestrutivo e permissivo aos interesses individuais dos Estados, em vez de estratégia para perseguir uma ambição internacionalmente determinada.

\section{CONSIDERAÇÕES FINAIS}

O Acordo de Paris segue uma lógica já anunciada no desmantelamento do tratamento diferenciado dos países em desenvolvimento em termos de obrigações centrais em outras searas do direito internacional - como o foi nos acordos comerciais no âmbito do GATT com o surgimento da OMC -, fazendo com que normas diferenciais desaparecessem dos tratados multilaterais, ao mesmo tempo em que normas contextuais são fortalecidas sob o argumento de que promovem mais diferenciação. Entretanto, o cotejo entre os acordos comerciais e os acordos ambientais, salientando que os últimos devam aderir ao mesmo tipo de diferenciação 'para todos' dos tratados comerciais, ignora, no caso das mudanças climáticas, que a atmosfera é um bem público global, o que não se pode afirmar do comércio internacional.

Sob a falsa promessa de prover 'mais diferenciação', o Acordo de Paris escamoteia as normas diferenciais consagradas na arquitetura do Protocolo de Quioto em nome de normas que permitem uma diferenciação contextual. Além disso, as obrigações contidas no Acordo, especialmente aquelas relacionadas à mitigação constante de seu artigo $4^{\circ}$, endossam de forma precisa e vinculante apenas obrigações de meio, obrigações procedimentais, não havendo nelas nenhuma orientação substancial para balizar as contribuições nacionalmente determinadas. Deste modo, a arquitetura jurídica de enfrentamento das mudanças climáticas no âmbito da CQMC modifica-se de uma estrutura normativa

76 RAJAMANI, Lavanya; BRUNNÉE, Jutta. The Legality of Downgrading Nationally Determined Contributions under the Paris Agreement: Lessons from the US Disengagement. Journal Of Environmental Law, [s...], v. 29, n. 3, p.537-551, 3 out. 2017. Oxford University Press (OUP). Disponível em: http://dx.doi.org/10.1093/jel/eqx024.

77 CULLET, P. Differential Treatment in Environmental Law: Addressing Critiques and Conceptualizing the Next Steps. Transnational Environmental Law, [s.l.], v. 5, n. 02, p.305-328, out. 2016. Cambridge University Press (CUP). Disponível em: http://dx.doi.org/10.1017/s204710251600025x. 
precisa e vinculante, com obrigações juridicamente densas para um modelo que, permeado apenas por obrigações de meio, os direitos internos passam a guiar o direito internacional, uma vez que os compromissos nacionais não são mais internacionalmente negociados.

Contudo, a principal derrota para os países em desenvolvimento que este modelo representa é que agora os compromissos de ações e reduções não são mais feitos na negociação internacional, com base em uma visão que tente ser a mais compartilhada possível de um mundo só. Nas negociações internacionais que os países em desenvolvimento sempre conseguiram expor as mazelas de um mundo injusto e induzir os países do Norte em direção a compromissos mais sérios. Porém, quando a discussão é levada para o âmbito doméstico dos Estados, essa pressão exercida pelos países desenvolvidos torna-se dispersa, quando não impossível de ser realizada.

Os países desenvolvidos devem continuar tomando a dianteira, liderando os esforços de enfretamento das mudanças climáticas, como bem afirma o artigo 4.3. Porém, de que modo? Nesse ponto, o Acordo é silente. Ou seja, não restou no âmbito do Acordo absolutamente nenhum mecanismo forte para que os países em desenvolvimento possam cobrar essa liderança dos países desenvolvidos em termos de obrigações centrais. O Acordo de Paris, de fato, põe de lado os conflitos distributivos, não os encerra, mas deixa o multilateralismo redistributivo dos países em desenvolvimento em posição marginal ou até mesmo sem espaço para se manifestar.

\section{REFERÊNCIAS}

BODANSKY, Daniel. The Paris Climate Change Agreement: A New Hope?. The American Journal Of International Law, [s.l.], v. 110, n. 2, p.288-319, 2016. American Society of International Law. Disponível em: http://dx.doi.org/10.5305/ amerjintelaw.110.2.0288.

CULLET, P. Differential Treatment in Environmental Law: Addressing Critiques and Conceptualizing the Next Steps. Transnational Environmental Law, [s.I.], v. 5, n. 02, p.305-328, out. 2016. Cambridge University Press (CUP). Disponível em: http://dx.doi.org/10.1017/s204710251600025x.

CULLET, P. Differential treatment in international law: towards a new paradigm of inter-state relations. European Journal Of International Law, [s.I], v. 10, n. 3, p.549-582, 1 mar. 1999. Oxford University Press (OUP). DOI: 10.1093/ ejil/10.3.549.

FALKNER, Robert. The Paris Agreement and the new logic of international climate politics. International Affairs, [s.I.], v. 92, n. 5, p.1107-1125, 31 ago. 2016. Oxford University Press (OUP). Disponível em: http://dx.doi.org/10.1111/1468-2346.12708.

FRENCH, Duncan. Developing States and International Environmental Law: The Importance of Differentiated Responsibilities. International \& Comparative Law Quarterly, [s.I], v. 49, n. 01, p.35-60, jan. 2000. Cambridge University Press (CUP). DOI: 10.1017/s0020589300063958.

HONKONEN, Tuula. The common but differentiated responsibility principle in multilateral environmental agreements: regulatory and policy aspects. Alphen aan den Rijn: Kluwer Law International, 2009.

KIELY, Ray. The rise and fall of emerging powers: Globalisation, US Power and the Global North-South divide. Londres: Palgrave Macmillian, 2016. (Global Reordering). 
MAGRAW, Daniel Barstow. Legal treatment of developing countries. Colorado Journal of International Environmental Law and Policy, Vol. 1, pp. 69-100, 1990.

MALJEAN-DUBOIS, Sandrine. The Paris Agreement: A New Step in the Gradual Evolution of Differential Treatment in the Climate Regime?. Review Of European, Comparative \& International Environmental Law, [s.l.], v. 25, n. 2, p.151-160, jul. 2016. Wiley-Blackwell. Disponível em: http://dx.doi.org/10.1111/reel.12162.

MCGEE, Jeffrey; STEFFEK, Jens. The Copenhagen Turn in Global Climate Governance and the Contentious History of Differentiation. Journal Of Environmental Law, [s.I.], v. 28, n. 1, p.37-63, mar. 2016. Oxford University Press (OUP). Disponivel em: http://dx.doi.org/10.1093/jel/eqw003.

O'NEIL, K. The Environment and International Relations. Cambridge: Cambridge University Press, 2009.

PARKS, Bradley C; ROBERTS, J Timmons. Inequality and the global climate regime: breaking the north-south impasse. Cambridge Review Of International Affairs, [s.I.], v. 21, n. 4, p.621-648, dez. 2008. Informa UK Limited. Disponível em: http://dx.doi.org/10.1080/09557570802452979.

PAUWELYN, Joost. The End of Differential Treatment for Developing Countries? Lessons from the Trade and Climate Change Regimes. Rev Euro Comp \& Int Env Law, [s.l], v. 22, n. 1, p.29-41, abr. 2013. Wiley-Blackwell. DOI: 10.1111/ reel.12017.

RAJAMANI, Lavanya. Ambition and Differentiation In The 2015 Paris Agreement: Interpretative Possibilities And Underlying Politics. International And Comparative Law Quarterly, [s.I.], v. 65, n. 02, p.493-514, 16 mar. 2016. Cambridge University Press (CUP). Disponível em: http://dx.doi.org/10.1017/s0020589316000130.

RAJAMANI, Lavanya. Differential Treatment in International Environmental Law. Oxford: Oxford University Press, 2006.

RAJAMANI, Lavanya; BRUNNÉE, Jutta. The Legality of Downgrading Nationally Determined Contributions under the Paris Agreement: Lessons from the US Disengagement. Journal of Environmental Law, [s.I.], v. 29, n. 3, p.537-551, 3 out. 2017. Oxford University Press (OUP). Disponível em: http://dx.doi.org/10.1093/jel/eqx024.

STONE, Christopher D. Common but Differentiated Responsibilities in International Law. The American Journal of International Law, [s.I], v. 98, n. 2, p.276-301, abr. 2004. JSTOR. DOI: 10.2307/3176729.

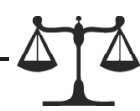

RECEBIDO EM: 21/07/2019

APROVADO EM: 18/11/2019 